\title{
Smart Nanostructured Materials: From Molecular Self- Assembly to Advanced Applications
}

\author{
Domenico Lombardo $\mathbb{D}^{1},{ }^{1}$ Mikhail A. Kiselev, ${ }^{2}$ Luigi Pasqua $\mathbb{D},{ }^{3}$ Giuseppe Pellicane $\mathbb{D},{ }^{4}$ \\ and Pietro Calandra (iD $^{5}$ \\ ${ }^{1}$ CNR-IPCF, Consiglio Nazionale delle Ricerche, Istituto per i Processi Chimico-Fisici, 98158 Messina, Italy \\ ${ }^{2}$ Frank Laboratory of Neutron Physics, Joint Institute for Nuclear Research, Dubna, Moscow, Russia \\ ${ }^{3}$ Dipartimento di Ingegneria Chimica e Ambientale, Università della Calabria, 87036-Rende (CS), Italy \\ ${ }^{4}$ University of KwaZulu-Natal, Durban, South Africa \\ ${ }^{5}$ CNR-ISMN, Consiglio Nazionale delle Ricerche, Istituto per lo Studio dei Materiali Nanostrutturati, 00015, \\ Monterotondo Stazione (RM), Italy \\ Correspondence should be addressed to Domenico Lombardo; lombardo@ipcf.cnr.it
}

Received 14 January 2021; Accepted 14 January 2021; Published 10 February 2021

Copyright (C) 2021 Domenico Lombardo et al. This is an open access article distributed under the Creative Commons Attribution License, which permits unrestricted use, distribution, and reproduction in any medium, provided the original work is properly cited.

The guest editors for this special issue are pleased to welcome contributions from researchers studying smart nanostructured materials developed from molecular self-assembly processes and their advanced applications. Molecular selfassembly can be exploited in key approaches for the design and development of nanostructured systems and has become a fundamental method for the formation of advanced nanomaterials. Manuscripts selected for this special issue deal with their product design, applications, and methods for the investigation of their structure and properties. A substantial number of papers were submitted, and after a thorough peer review process, six papers were selected to be included in this special issue. These papers cover a wide range of novel materials for advanced applications. We believe that the papers collected in this special issue highlight current relevant topics in research related to the molecular self-assembly of nanomaterials and their advanced applications and will introduce readers to important advances in this field.

$\mathrm{N}$. Fu et al. [1] investigated the influence of different molar ratios of Ti/Si on the morphology, structure, and photocatalytic activity of core-shell $\mathrm{SiO}_{2} @ \mathrm{TiO}_{2}$ nanoparticles (CSTNs) for photocatalytic applications. A high photocatalytic efficiency of the CSTNs (synthesized by means of a hydrothermal method) was shown with low photocatalyst addition by studying the photocatalytic activity of the CSTNs for both colourless organic pollutants (such as phenol) and coloured substances (such as methylene blue) under UV irradiation. More specifically, the study evidences that the CSTNs with the Ti/Si molar ratio of $5: 1$ had the highest photocatalytic activity of $99.4 \%$ towards phenol and $99.2 \%$ towards methylene blue, which showed higher photocatalytic efficiency with the addition of $0.25 \mathrm{~g} / \mathrm{L}$ photocatalyst compared with the other reported $\mathrm{TiO}_{2}-\mathrm{SiO}_{2}$ composites.

The review article by M. M. Rahman et al. [2] furnished an up-to-date documentary on various catalysts used for the oxidation of alkyl-substituted benzenes along with their reaction conditions and selectivity profiles. Because of the substantial improvements in product yield and catalyst reusability, heterogeneous catalysts have gained growing consideration in recent years. In this respect, this review article work updates our knowledge for the selection and/or design of novel catalysts for chemists and engineers in industrial and academic settings.

In the paper by X. Wang et al. [3], nano-BiOBr photocatalysts were successfully prepared by the hydrothermal method, while the influences of different solvents (and concentrations) on the structure, morphology, optical properties, and photocatalytic properties were investigated systematically. The 
results indicated that the concentration and solvents have an essential influence on the bandgap energy values of the nano$\mathrm{BiOBr}$ photocatalyst, and the photocatalyst showed an excellent activity on the photodegradation of rhodamine $B$. Finally, the high photocatalytic activity of photocatalysts for contaminant aqueous solutions investigated in this paper may stimulate the development of new platforms to develop flexible photocatalysts for practical applications in water purification.

In the article by N. T. V. Hoan et al. [4], the synthesis of $\mathrm{TiO}_{2} /$ diazonium/graphene oxide composites and their application to visible-light-driven photocatalytic degradation of methylene blue (MB) in a visible region have been studied. The photocatalytic oxidation exhibited a complete decomposition of MB by the cleavage and oxidation of one or more of the methyl substituents on the amine groups. The catalyst was stable and recyclable after three times with a negligible loss of catalytic activity. In addition, the $\mathrm{TiO}_{2}$ /diazonium/graphene oxide can also photocatalyze for the degradation of some other dyes (phenol, methyl red, and Congo red). To explain the experimental behaviour, the authors proposed a kinetic model by the combination of the classical Langmuir-Hinshelwood kinetic model and Langmuir isotherm model. Finally, the proposed catalyst with its stability and recyclable advantages is promising for application in the treatment of dye wastes.

In the study by L. A. Kaledin et al. [5], the aluminumwater reaction has successfully been used to produce $2 \mathrm{D}$ and $1 \mathrm{D}$ quantum (and nanometer) size of $\gamma$-AlOOH (Boehmite) and $\beta$-FeOOH (Akaganeite) $/ \gamma$-AlOOH (Boehmite) mesostructures deposited onto siliceous substrates in a one-step process at moderate temperature. The investigation of the role of surface nanoscale roughness and charging behaviour evidenced that the isoelectric point values of rough nanostructured surfaces were three $\mathrm{pH}$ units higher compared to the flat crystalline $\gamma-\mathrm{AlOOH}$ (Boehmite) and $\beta$ $\mathrm{FeOOH}$ (Akaganeite) surfaces, resulting in a high removal efficacy of submicron particles from aqueous suspension by the surfaces with combined microscale and nanoscale structures. The authors also proposed a model explaining the $\mathrm{pH}$-dependent behaviour of the zeta potential and suggested the existence of a coupling electrokinetic effect of the local electrical double layer (EDL) fields with the local flow fields.

Finally, in the review paper by C. Cretu et al. [6], the basic concepts on self-assembly of small amphiphilic molecules with noble metals are shown with reference to their photophysical properties. The article is aimed at furnishing to the reader a panoramic view of this exciting problematics in terms of (i) the principles of self-assembly of amphiphiles that involve noble metals, (ii) the preparation of opportune amphiphilenoble metal hybrids, and (iii) examples of amphiphile-noble metal systems as representative of systems with enhanced photophysical properties. Finally, comments and perspectives are given with special attention to a range of possible modern and advanced applications in nanotechnology.

The six articles of this special issue will stimulate readers' creativity around the possible development of this research area. Moreover, although these six papers cannot provide the full image of the field of advanced self-assembled nano- materials, we nevertheless hope that the combination of these contributed papers will inspire the readers to undertake their research work in this very wide area of materials investigation.

\section{Conflicts of Interest}

The editors declare that they have no conflicts of interest regarding the publication of this special issue.

\section{Acknowledgments}

The editors would like to thank all authors for having submitted their manuscripts to this special issue, the editors for having spent their time selecting reviewers and making editorial decisions, and of course the reviewers for providing their expert opinions.

Domenico Lombardo
Mikhail A. Kiselev
Luigi Pasqua
Giuseppe Pellicane
Pietro Calandra

\section{References}

[1] N. Fu, X.-c. Ren, and J.-x. Wan, "The effect of molar ratios of $\mathrm{Ti} / \mathrm{Si}$ on core-shell $\mathrm{SiO}_{2} @ \mathrm{TiO}_{2}$ nanoparticles for photocatalytic applications," Journal of Nanomaterials, vol. 2020, Article ID 5312376, 11 pages, 2020.

[2] G. Ara, S. Rahman, S. Uddin, M. N. Bin-Jumah, and M. M. Abdel-Daim, "Recent development of catalytic materials for ethylbenzene oxidation," Journal of Nanomaterials, vol. 2020, Article ID 7532767, 20 pages, 2020.

[3] X. Wang, F. Zhang, Y. Yang, Y. Zhang, L. Liu, and W. Lei, "Controllable synthesis and photocatalytic activity of nano-BiOBr photocatalyst," Journal of Nanomaterials, vol. 2020, Article ID 1013075, 7 pages, 2020.

[4] N. T. V. Hoan, N. N. Minh, T. T. K. Nhi et al., " $\mathrm{TiO}_{2} /$ diazonium/graphene oxide composites: synthesis and visible-lightdriven photocatalytic degradation of methylene blue," Journal of Nanomaterials, vol. 2020, Article ID 4350125, 15 pages, 2020.

[5] L. A. Kaledin, F. Tepper, Y. Vesga, and T. G. Kaledin, "Boehmite and akaganeite $1 \mathrm{D}$ and $2 \mathrm{D}$ mesostructures: synthesis, growth mechanism, ageing characteristics and surface nanoscale roughness effect on water purification," Journal of Nanomaterials, vol. 2019, Article ID 9516156, 10 pages, 2019.

[6] C. Cretu, L. Maiuolo, D. Lombardo, E. I. Szerb, and P. Calandra, "Luminescent Supramolecular Nano- or Microstructures Formed in Aqueous Media by Amphiphile-Noble Metal Complexes," Journal of Nanomaterials, vol. 2020, 24 pages, 2020. 